Sharif University of Technology
Scientia Iranica

\title{
Dynamics and circuit simulation of a fractional-order hyperchaotic system
}

\author{
A. Lassoued ${ }^{a}$, F. Nazarimehr ${ }^{b, *}$, and O. Boubaker ${ }^{a}$ \\ a. University of Carthage, National Institute of Applied Sciences and Technology, INSAT, Centre Urbain Nord, BP676, 1080 Tunis \\ Cedex, Tunisia. \\ b. Department of Biomedical Engineering, Amirkabir University of Technology, Tehran, P.O. Box 15875-4413, Iran.
}

Received 18 January 2020; received in revised form 17 March 2021; accepted 19 July 2021

\author{
KEYWORDS \\ Fractional-order \\ derivatives; \\ Hyperchaotic systems; \\ Circuit design; \\ Charef approximation; \\ Fractional-order \\ polynomial.
}

\begin{abstract}
A hyperchaotic system with fractional terms and fractional-order derivatives is investigated in this paper. Simulations show that different attractors such as equilibrium point, limit cycle, and hyperchaotic attractor can be generated by the system. Circuit of fractional-order integrator is designed and used to implement the circuit of the studied system. The circuit implementation of the studied system proves its feasibility.
\end{abstract}

(C) 2023 Sharif University of Technology. All rights reserved.

\section{Introduction}

Fractional calculus has been an interesting topic since the 17th century. There are different definitions for fractional-order derivative. Three common methods for calculation of fractional-order derivative are Grunwald-Letnikov, Riemann-Liouville, and Caputo definitions [1-3]. Fractional calculus has many applications in different areas such as physics and chemical engineering [4]. Chaotic dynamics are very complex and several chaotic and hyperchaotic systems have been discovered over the last decades [5]. Chaotic attractors have only one Lyapunov Exponent (LE) where hyperchaotic attractors have more than one positive LE. Thus, they have more complex dynamic systems than chaotic ones. The generation of hyperchaotic attractors of dynamical systems is not well known

\footnotetext{
*. Corresponding author.

E-mail addresses: lassoued.abir5@gmail.com (A. Lassoued); fahimenazarimehr@yahoo.com (F. Nazarimehr); olfa.boubaker@insat.rnu.tn (O.Boubaker)
}

for researchers. Fractional-order systems are more complex than integer orders. Therefore, the study of fractional-order hyperchaotic is critical. Fractionalorder systems such as fractional-order Lorenz system [6] and fractional-order system without any equilibrium point [7] have been proposed recently. The time series of a hyperchaotic system is highly dependent on initial conditions. It is implied that a small difference in the initial conditions causes a huge difference in the final states of hyperchaotic systems.

Circuit implementation of chaotic systems is very important since it realizes the feasibility of their dynamics. Implementation of chaotic dynamics has been a hot topic for many years [8-10]. Feasibility of fractional-order systems has been studied in many works [11]. Circuit implementation of a fractionalorder system based on Chen system was investigated in [11]. In [12], a novel jerk chaotic system and its circuit implementation were developed.

The paper suggests a fractional-order system with more complex dynamics than those proposed in recent papers. In this system, fractional-order derivatives and fractional-order polynomial are combined. To enhance the potential application of the investigated system, 
its electronic circuit is then implemented to prove its feasibility.

\section{Fractional calculus: Basic proprieties}

In this paper, the Caputo is the adopted definition for the calculation of fractional-order derivatives. This method is used in engineering applications.

Definition 1: The Caputo approach is defined as [13]:

$$
D^{q} x(t)=J^{m-q} x^{(m)}(t), \quad q>0,
$$

where $D^{q}$ is the noninteger-order differ-integral operator. $q$ is the fractional-order and $m$ is its integer part. $J$ is the integral operator described as:

$$
J^{\beta} x(t)=\frac{1}{\Gamma(\beta)} \int_{0}^{\infty}(t-\sigma)^{\beta-1} y(\sigma) d \sigma .
$$

$\Gamma(\beta)$ is the Gamma function [13].

The stability analysis of fractional-order systems is more complex than the integer orders which need particular definitions, as introduced below.

Definition 2: Let us consider the following fractional-order model [14]:

$$
\frac{d^{q} x(t)}{d t^{q}}=f(x(t))
$$

where $0<q<1$ and $x \in \mathbb{R}^{n}$. As shown in [15], the equilibrium points of $f(x(t))$ are locally asymptotically stable if all eigenvalues $\lambda_{i}$ of the Jacobian matrix $J=\partial f(x(t)) / \partial x(t)$ evaluated at the equilibrium points satisfy $\left|\arg \left(\lambda_{i}\right)\right|>q \frac{\pi}{2}$.

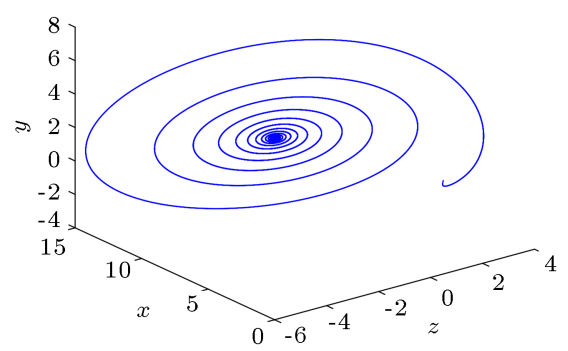

(a)

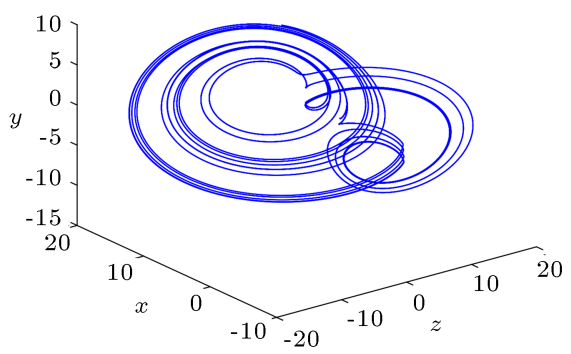

(c)

\section{A fractional-order hyperchaotic system}

A hyperchaotic system with both fractional-order derivatives and terms is investigated. The designed system is based on the recently proposed system introduced in [16]. By modifying the derivative operator, the designed system is as follows:

$$
\left\{\begin{array}{l}
D^{q} x=y \\
D^{q} y=z \\
D^{q} z=-a z-b y+G(x) \\
D^{q} w=k y-h w+G(x)
\end{array}\right.
$$

where $0<q<1$. $G(x)$ is a nonlinear function defined as:

$$
G(x)= \begin{cases}(-c-d) x^{2}-m(-x)^{r-1}, & \text { if } x<0 \\ 0, & \text { if } x=0 \\ (-c+d) x^{2}+m(x)^{r-1}, & \text { if } x>0 .\end{cases}
$$

$(a, b, c, d, k, h, m, r)$ are the system parameters. $r$ is a fractional number in the interval $1<r<2$. Parameters $(a, b, c, d, k, h, m, r)$ are set to $(0.93,1.11$, $-0.11,-0.21,0.001,14,6.26,1.3)$. By varying the parameter $q$, System (4) exhibits different attractors as in the following:

(i) If $q \leq 0.96$, regular attractors are obtained;

(ii) If $q \in[0.96 \cdots 1]$, strange attractors are obtained.

Phase portraits of different attractors are shown in Figure 1. If $q=1$, System (4) will be equivalent to the hyperchaotic system presented in [16]. When

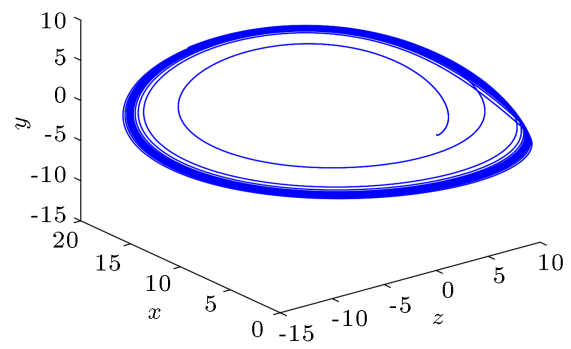

(b)

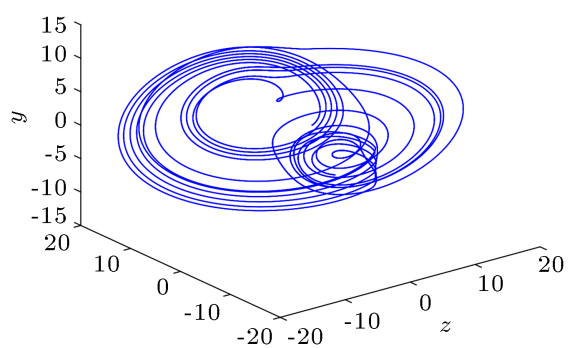

(d)

Figure 1. Regular and strange attractors of System (4): (a) $q=0.88$, (b) $q=0.96$, (c) $q=0.97$, and (d) $q=0.99$. 


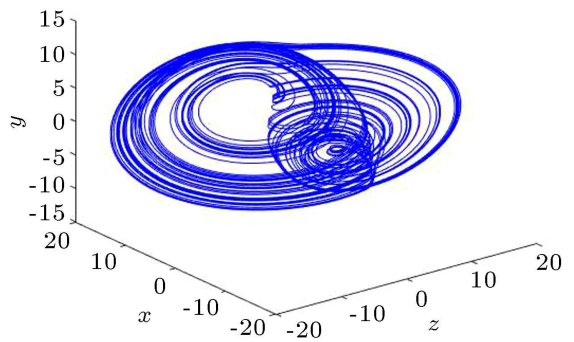

(a)

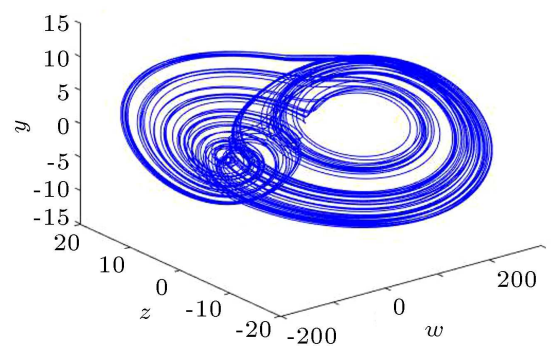

(b)

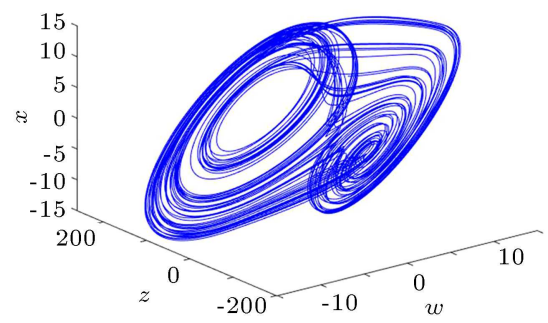

(c)

Figure 2. Projections of the attractor, related to the hyperchaotic System (4) when $q=0.97$ in (a) $x y z$, (b) wyz, and (c) $w x z$ space.

$q=0.98$, a strange attractor is observed, as shown in Figure 2. This attractor is asymmetric and is characterized by two scrolls with different sizes.

Sensitivity to initial conditions is still valid for fractional-order hyperchaotic systems. Figure 3 represents the time evolution of state variables $x, y, z$, and $w$ with two initial conditions $(1,1,1,1)$ and $\left(1+10^{-6}\right.$, 1, 1, 1). Figure 4 shows the errors between the two time series of each state variable. We have deduced that the two trajectories of System (4) for each variable are initially identical, but they completely differ after a certain time. It is clear that System (4) is sensitive to initial conditions.

System (4) exhibits four LEs such as $L E_{1}=0.210$, $L E_{2}=0.026, L E_{3}=0$, and $L E_{4}=-1.199$. These LEs are estimated using the MATLAB code for LEs of fractional-order systems described in [17]. Since the LE spectrum has two positive LEs, System (4) is hyperchaotic. $L E_{1}$ is the largest positive one and this exponent increases the expansion degree of the fractional-order attractor in the phase space.

The equilibrium points of System (4) are the roots of these equations:

$$
y=z=w=0, \quad G(x)=0 .
$$

Based on [16], the following results are obtained:

(i) If $x=0$, then $H_{1}=(0,0,0,0)$ is an equilibrium point of System (4);

(ii) If $x>0$, then $H_{2}=\left(((c-d) / m)^{1 / \alpha}, 0,0,0\right)$ is an equilibrium point of System (4) when $\alpha=r-3$;

(iii) If $x<0$, then $H_{3}=\left((-(-c-d) / m)^{1 / \alpha}, 0,0,0\right)$ is an equilibrium point of System (4) when $\alpha=r-3$.
As a result, the hyperchaotic system admits three asymmetric equilibrium points. By considering the mentioned parameters, three algebraic points are obtained as $H_{1}=(0,0,0,0), H_{2}=(11.73,0,0,0)$, and $H_{3}=(-5.87,0,0,0)$. To analyze the stability of each equilibrium point, Jacobian matrix should be computed. Table 1 summarizes eigenvalues of each equilibrium point of System (4). An argument is determined for each eigenvalue, noted $\arg \left(\lambda_{i}\right)$.

Based on Definition 2, System (4) can exhibit a strange attractor only when it has at least one unstable equilibrium point. More precisely, at least one of its equilibrium points must satisfy the following condition:

$$
q<\frac{2}{\pi}\left|\arg \left(\lambda_{i}\right)\right|, \quad q<\frac{2}{\pi} \arctan \left(\frac{\left|\operatorname{Im}\left(\lambda_{i}\right)\right|}{\left|\operatorname{Re}\left(\lambda_{i}\right)\right|}\right),
$$

where $i=(1, \cdots, 4) . \quad \operatorname{Im}\left(\lambda_{i}\right)$ and $\operatorname{Re}\left(\lambda_{i}\right)$ denote the imaginary and real parts of the eigenvalues $\lambda_{i}$, respectively. To illustrate Condition (7), Figure 5 presents the stable and unstable regions of System (4) in $q=0.97$.

Referring to Table 1 , it is easy to say that for the first equilibrium point $H_{1}, \lambda_{1}$ belongs to the stable region while $\lambda_{2}, \lambda_{3}$, and $\lambda_{4}$ belong to the unstable region. However, for the equilibrium points $H_{2}$ and $H_{3}, \lambda_{1}$ and $\lambda_{2}$ belong to the unstable region, while $\lambda_{3}$ and $\lambda_{4}$ belong to the stable region.

\section{The fractional-order integrator}

4.1. Approximation of the fractional derivative Based on the Caputo definition and assuming that the initial conditions are null, the Laplace transform 


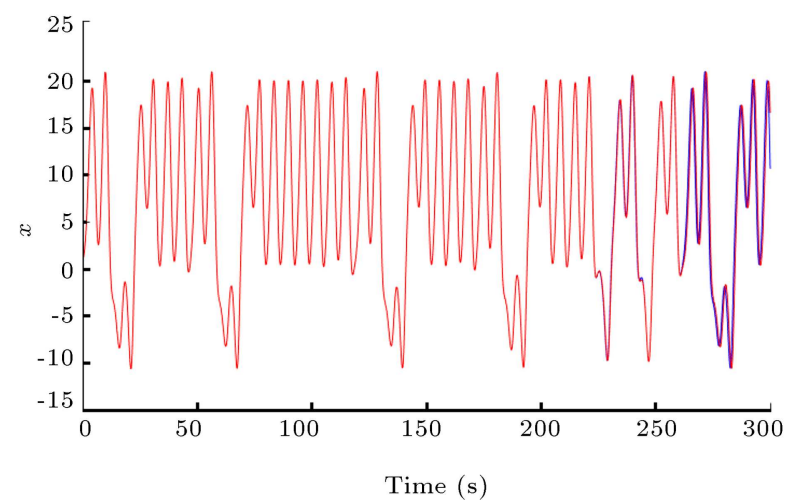

(a)

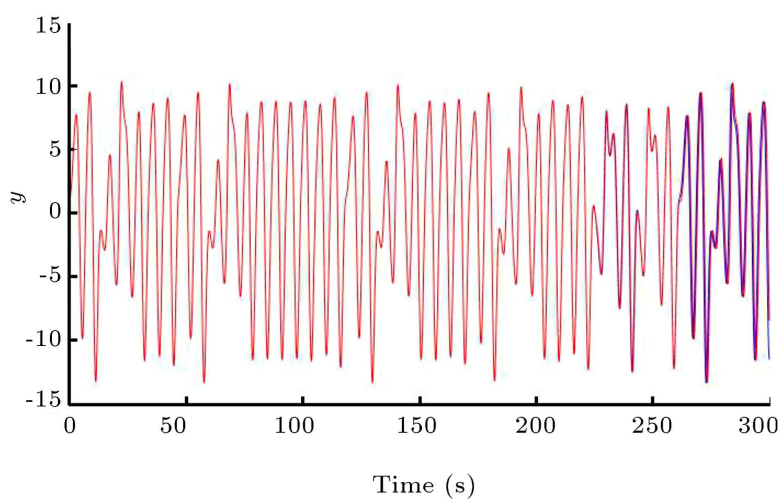

(b)

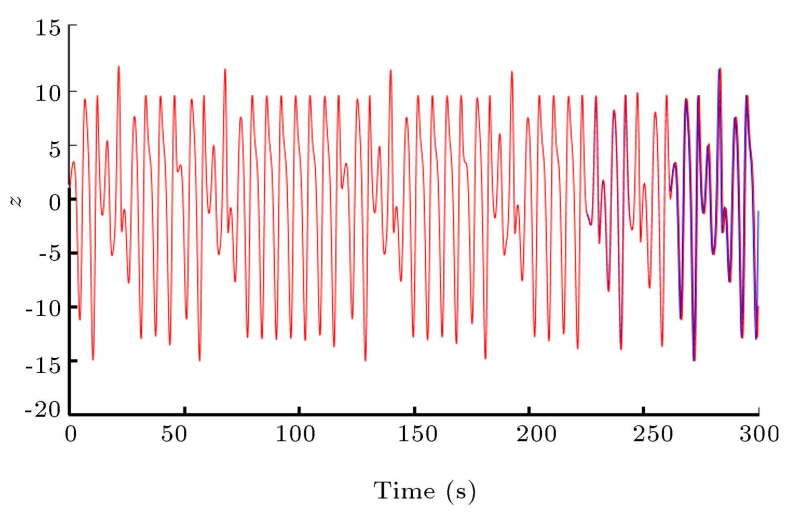

(c)

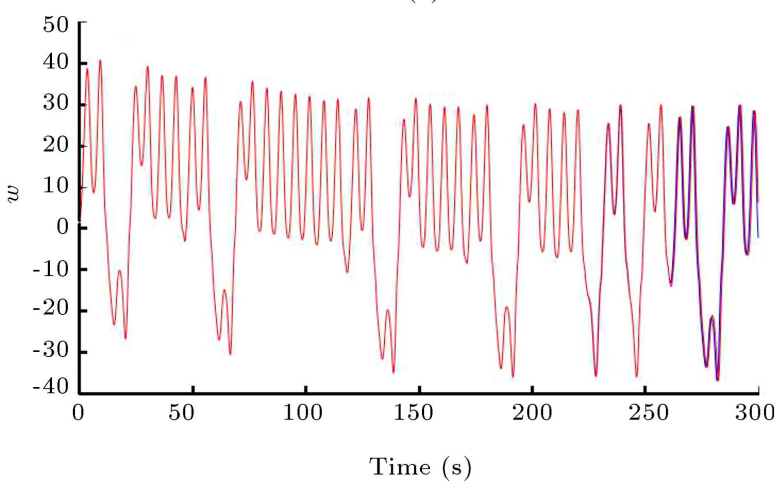

(d)

Figure 3. Time series of System (4) with initial conditions $(1,1,1,1)$ (blue) and $\left(1+10^{6}, 1,1,1\right)$ (red): (a) $x$, (b) $y,(\mathrm{c}) z$, and (d) $w$.

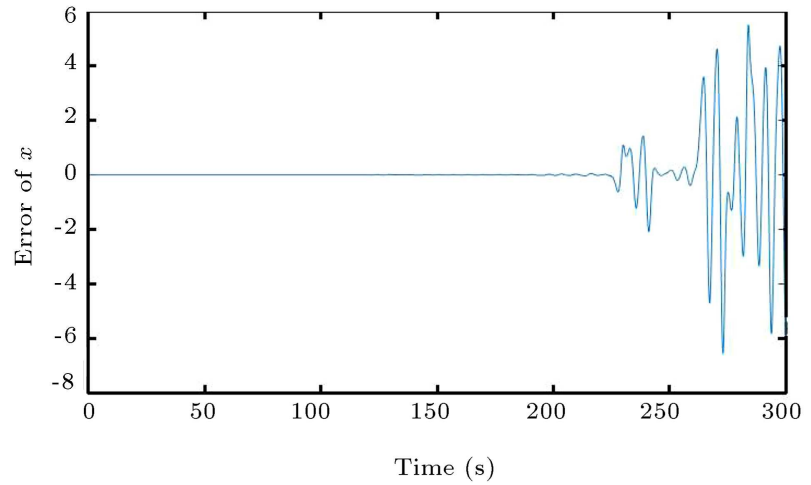

(a)

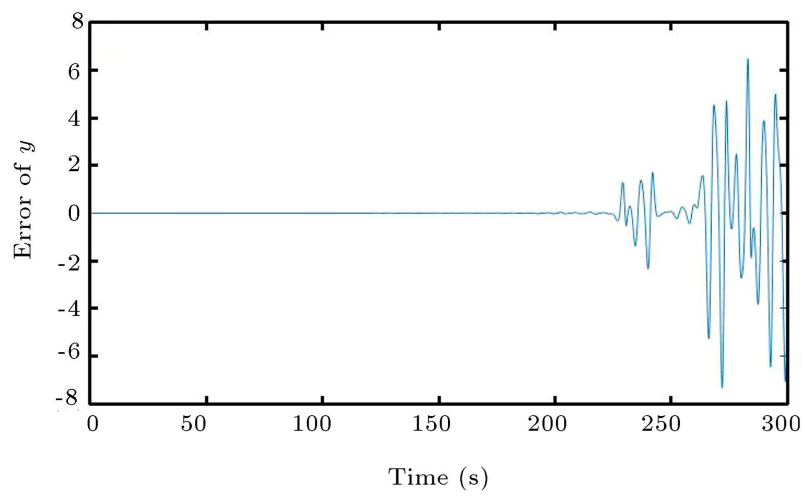

(b)

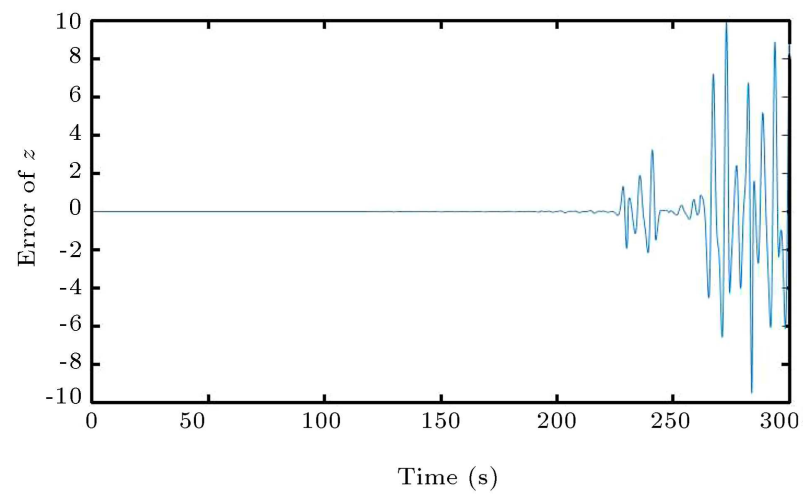

(c)

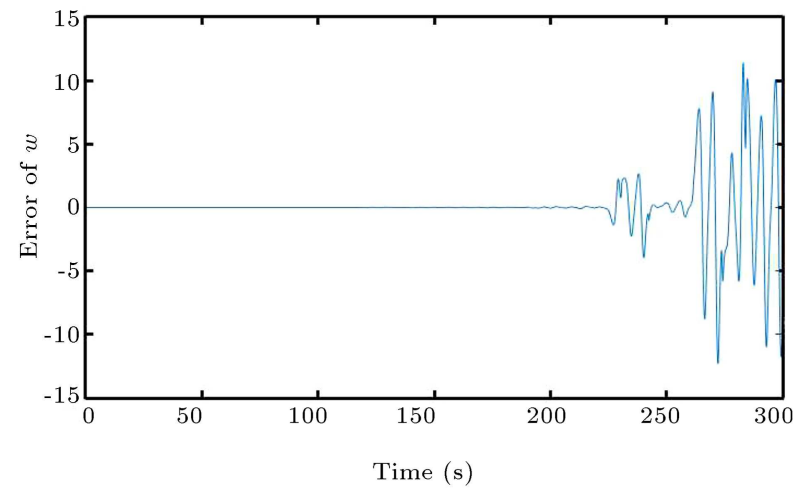

(d)

Figure 4. Error between the trajectories with the initial conditions $(1,1,1,1)$ and $\left(1+10^{-6}, 1,1,1\right)$ : (a) $x$, (b) $y$, (c) $z$, and (d) $w$. 
Table 1. Stability analysis of the hyperchaotic System (4).

\begin{tabular}{cll}
\hline Equilibrium points & \multicolumn{1}{c}{ Eigenvalues } & \multicolumn{1}{c}{$\arg \left(\boldsymbol{\lambda}_{\boldsymbol{i}}\right)$} \\
\hline & $\lambda_{1}=0$ & $\arg \left(\lambda_{1}\right)=0$ \\
$\lambda_{2}=-0.001$ & $\arg \left(\lambda_{2}\right)=\pi$ \\
$\lambda_{3}=-0.465+0.945 i$ & $\arg \left(\lambda_{3}\right)=2.027$ \\
$\lambda_{4}=-0.465-0.945 i$ & $\arg \left(\lambda_{4}\right)=-2.027$ \\
& & \\
& $\lambda_{1}=-0.001$ & $\arg \left(\lambda_{1}\right)=\pi$ \\
& $\lambda_{2}=-1.738$ & $\arg \left(\lambda_{2}\right)=\pi$ \\
& $\lambda_{3}=-0.404+1.533 i$ & $\arg \left(\lambda_{3}\right)=1.313$ \\
& $\lambda_{4}=-0.404-1.533 i$ & $\arg \left(\lambda_{4}\right)=-1.313$ \\
$\Delta_{2}=2 d x-2 c x+(r-1) m x^{r-2}$ & \\
& & \\
& $\lambda_{1}=-0.001$ & $\arg \left(\lambda_{1}\right)=\pi$ \\
& $\lambda_{2}=-1.193$ & $\arg \left(\lambda_{2}\right)=\pi$ \\
& $\lambda_{3}=-0.131+1.186 i$ & $\arg \left(\lambda_{3}\right)=1.460$ \\
& $\lambda_{4}=-0.131-1.186 i$ & $\arg \left(\lambda_{4}\right)=-1.460$ \\
& $\Delta_{2}=-2 d x-2 c x-(r-1) m x^{r-2}$ \\
\hline
\end{tabular}

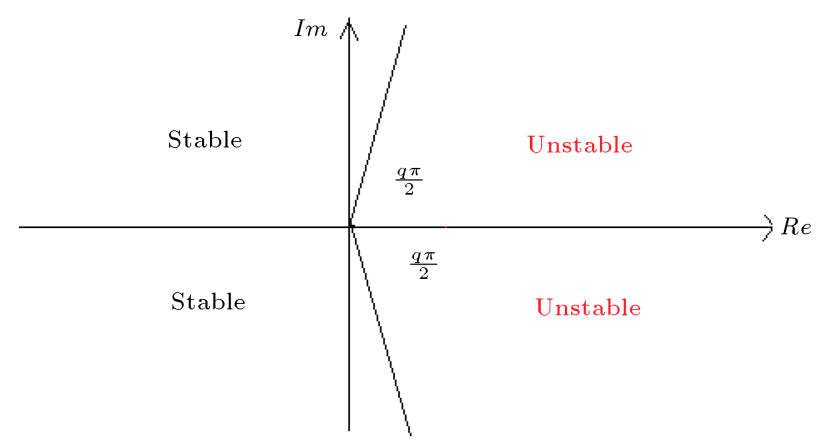

Figure 5. Stable and unstable regions of System (4) in $q=0.97$.

of the fractional derivative is defined by the following equation [13]:

$$
\mathscr{L}\left\{D^{q} x(t)\right\}=S^{q} \mathscr{L}\{x(t)\} .
$$

Thus, the fractional-order integration $q$ is represented by the transfer function $F(s)=\frac{1}{s^{q}}$ in the fractional domain, where $s$ is the Laplace operator. To reduce the calculation's complexity, $F(s)$ is approximated by an integer-order transfer function. There are many methods based on the concept of Fractional Power Poles (FPP). They are called the approximation method with $n$ zeros and $n$ poles or $n$ zeros and $n-1$ poles. The most famous one is the approximation of Charef et al. [18]. The Charef approximation allows a linear approximation in the frequency domain. Specifically, the approximation of the fractional-order integrator $\left(\frac{1}{s^{q}}\right)$ depends on the desired bandwidth and the amplitude error between the Bode diagrams before and after approximation.
Theorem 1 [18]. The Charef approximation is defined as follows:

$$
\frac{1}{s^{q}} \simeq \frac{1}{\left(1+\frac{s}{p_{T}}\right)^{q}} \simeq \frac{\prod_{i=0}^{N-1}\left(1+\frac{s}{Z_{i}}\right)}{\prod_{i=0}^{N}\left(1+\frac{s}{P_{i}}\right)}
$$

where $N$ is the approximation order and $q$ is the fractional-order of $F(s) . P_{i}$ and $Z_{i}$ are the poles and zeros of the Charef approximation. $\frac{1}{p_{T}}$ is the relaxation time constant. According to this method, $P_{i}$ and $Z_{i}$ are defined as follows:

$$
\begin{aligned}
& P_{0}=P_{\alpha} \sqrt{b}, \quad P_{i}=P_{0}(a b)^{i}, \quad i=1, \cdots, N, \\
& Z_{i}=P_{0} a(a b)^{i}, \quad i=0, \cdots, N-1,
\end{aligned}
$$

where $P_{\alpha}$ is the selected limited frequency. $a$ is the location rate of one zero relative to its previous pole and $b$ is the location rate of one pole relative to its previous zero. These two rates are calculated as follows:

$$
a=10^{\left(\frac{Y}{10(1-q)}\right)}, \quad b=10^{\left(\frac{Y}{10 q}\right)},
$$

where $Y$ is the approximation error expressed in $\mathrm{dB}$.

The approximation order $N$ is calculated by the following definition:

$$
N=\operatorname{integer}\left(\frac{\log \left(\frac{w_{\max }}{P_{0}}\right)}{\log (a b)}\right)+1,
$$

where $w_{\max }$ is the corresponding pulse to the maximum error. 
Table 2. Coordinate points of $L_{1}, L_{2}$, and $L_{3}$ for the two fractional-order transfer functions.

\begin{tabular}{ccc}
\hline Points & $\begin{array}{c}\text { Amplitude dB } \\
\text { (transfer function before approximation) }\end{array}$ & $\begin{array}{c}\text { Amplitude dB } \\
\text { (transfer function after approximation) }\end{array}$ \\
\hline$H_{1}$ & -0.4 & -2.71 \\
$H_{2}$ & -58.5 & -59.1 \\
$H_{3}$ & -116 & -116 \\
\hline
\end{tabular}

In the literature, several fractional-order integrators have been approximated by the Charef method. There is a summary table of these orders ranging from 0.1 to 0.9 with a step 0.1 and an approximation error $1 \mathrm{~dB}$. This table was presented in the reference [19]. System (4) exhibits a strange attractor where $q \in$ $[0.96 \cdots 1]$. Thus, the function $\frac{1}{s^{0.97}}$ corresponding to the fractional derivative $D^{0.97}$ should be approximated. Based on new works in the literature, we did not find the corresponding approximation to $q=0.97$. To apply the Charef method, the parameters $\left(P_{\alpha}, w_{\max }, Y\right)$ are fixed to $\left(10^{-3}, 1000,1 \mathrm{~dB}\right) . P_{\alpha}$ and $w_{\max }$ are the limit frequencies of the approximation. Using the MATLAB software, the obtained results are:

$$
\begin{array}{lll}
N=2, & a=2154.4346, & b=1.2679, \\
P_{0}=0.0011, & P_{1}=3.0759, & P_{2}=8402.3085, \\
Z_{0}=2.4259, & Z_{1}=6626.8301 . &
\end{array}
$$

It is an approximation of order 2 (two zeros and three poles). The obtained transfer function is described as follows:

$$
\begin{aligned}
\frac{1}{s^{0.97}} & =\frac{\left(1+\frac{s}{Z_{0}}\right)\left(1+\frac{s}{Z_{1}}\right)}{\left(1+\frac{s}{P_{0}}\right)\left(1+\frac{s}{P_{1}}\right)\left(1+\frac{s}{P_{2}}\right)} \\
& =\frac{P_{0} P_{1} P_{2}}{Z_{0} Z_{1}} \frac{\left(s+Z_{0}\right)\left(s+Z_{1}\right)}{\left(s+P_{0}\right)\left(s+P_{1}\right)\left(s+P_{2}\right)} \\
& =\frac{0.018 s^{2}+12 s+29.1}{s^{3}+8405.4 s^{2}+25854 s+28.4291} .
\end{aligned}
$$

In order to verify the accuracy of the obtained results, the Bode magnitude diagram for the 0.97 fractional integrator and its approximated transfer function is realized using the FOMCOM toolbox and the predefined functions under MATLAB software. The obtained results are shown in Figure 6. The two obtained Bode diagrams are similar. Since the two simulations are conducted with different methods (FOMCOM toolbox and MATLAB algorithm), it is difficult to group them on the same graph. To remedy this problem, we identified the coordinates of three specific points on the two trajectories. These points are $L_{1}, L_{2}$, and $L_{3}$ corresponding to the frequencies $10^{-3}, 1$, and

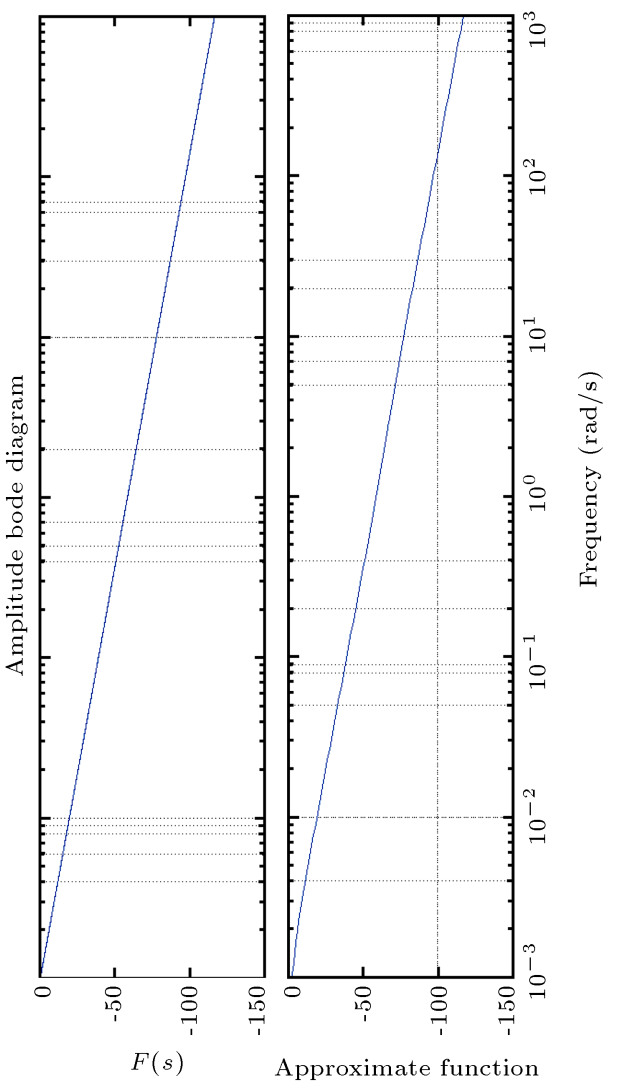

Figure 6. Magnitude bode diagram of fractional-order transfer function before and after approximation with $q=0.97$.

$10^{-3}(\mathrm{rad} / \mathrm{s})$, respectively. Table 2 summarizes these coordinates.

Figure 6 and Table 2 prove that the Charef method gives very good approximation results with a margin of error $1 \mathrm{~dB}$ in the frequency range $\left[P_{\alpha}, w_{\max }\right]=\left[10^{-3}, 10^{3}\right]$.

\subsection{Circuit design of the fractional-order integrator}

To realize the fractional-order approximation of $(N+1)$ poles and $N$ zeros, several circuits are used. Figure 7 represents one of the widely used circuit to design the approximate transfer function $\frac{1}{s^{q}}$ based on Charef approximation [20]. $n$ is equal to the number of poles.

In our case, the number of poles is $N+1=3$. The designed circuit contains three resistors and three capacitors. Its corresponding transfer function $H(s)$ 


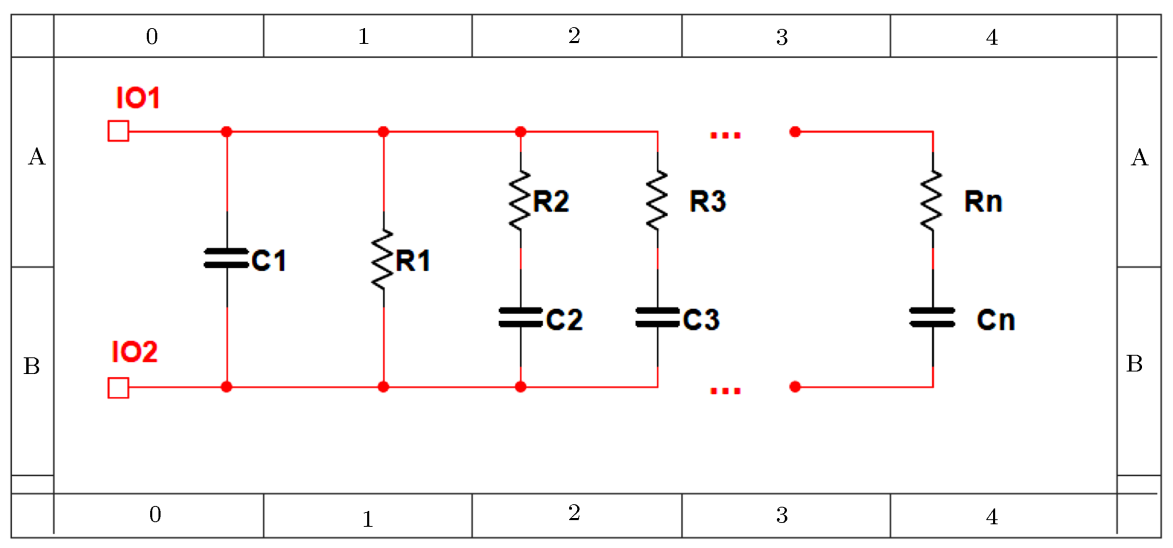

Figure 7. Circuit of the approximate function $\frac{1}{s^{m}}$ based on Charef approximation.

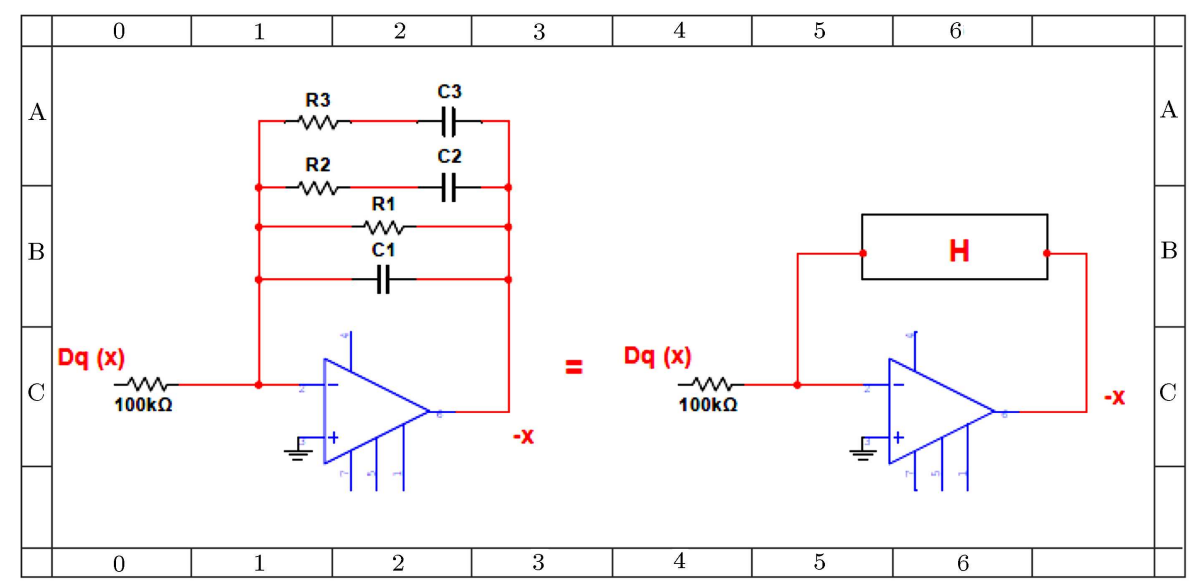

Figure 8. Circuit design of $H(s)$ in $q=0.97$.

when $q=0.97$ is defined as follows:

$$
\begin{aligned}
H(s) & =R_{1} / / \frac{1}{s C_{1}} / /\left(R_{2}+\frac{1}{s C_{2}}\right) / /\left(R_{3}+\frac{1}{s C_{3}}\right) \\
& =\frac{\frac{1}{C_{1}}\left(s+\frac{1}{R_{2} C_{2}}\right)\left(s+\frac{1}{R_{3} C_{3}}\right)}{s^{3}+\alpha s^{2}+\beta s+\gamma},
\end{aligned}
$$

with:

$$
\begin{aligned}
& \alpha=\frac{\left(R_{1} C_{1}+R_{2} C_{2}+R_{1} C_{2}\right) R_{3} C_{3}+R_{1} R_{2} C_{2}\left(C_{1}+C_{3}\right)}{R_{3} R_{2} R_{1} C_{1} C_{2} C_{3}}, \\
& \beta=\frac{R_{1} C_{1}+R_{2} C_{2}+R_{1} C_{2}+R_{1} C_{3}+R_{3} C_{3}}{R_{3} R_{2} R_{1} C_{1} C_{2} C_{3}}, \\
& \gamma=\frac{1}{R_{3} R_{2} R_{1} C_{1} C_{2} C_{3}} .
\end{aligned}
$$

Using Eqs. (13) and (14), the numerical values are obtained:

$$
\begin{array}{ll}
R_{1}=10.29 \mathrm{k} \Omega, & R_{2}=293.70 \mathrm{k} \Omega, \\
R_{3}=9.6 \mathrm{k} \Omega, & C_{1}=0.5524 \mathrm{nF},
\end{array}
$$

$$
C_{2}=0.1396 \mathrm{nF}, \quad C_{3}=0.1 \mathrm{nF} .
$$

Figure 8 describes the circuit of the fractional-order integrator 0.97 with Multisim software.

\section{Circuit design}

\subsection{Reduced system}

To implement the non-integer order terms in System (4), we have designed a specific case study when the system parameter $r$ is fixed to 1.5. Thus, the studied system is defined by the following differential equations:

$$
\left\{\begin{array}{l}
D^{q} x=y \\
D^{q} y=z \\
D^{q} z=-a z-b y-c x^{2}+d|x| x+m \sqrt{|x|} \operatorname{sgn}(x) \\
D^{q} w=k y-h w-c x^{2}+d|x| x+m \sqrt{|x|} \operatorname{sgn}(x)
\end{array}\right.
$$

where the parameters $(a, b, c, d, m, h, k, q)$ are equal to $(1,1,-0.11,-0.21,5,0.01,1.4,0.97)$. The strange attractors are presented in Figure 9. Based on Figure 9, the maximum and minimum values of the signals 


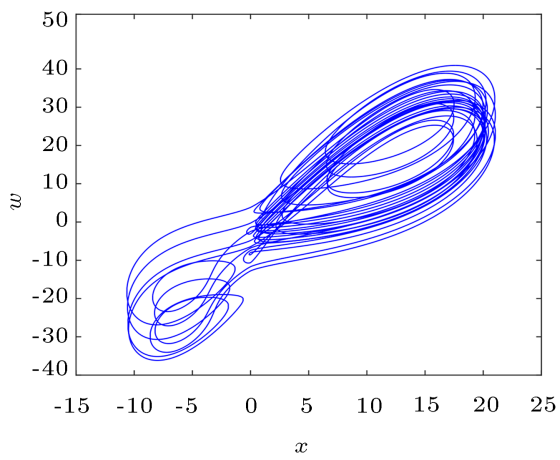

(a)

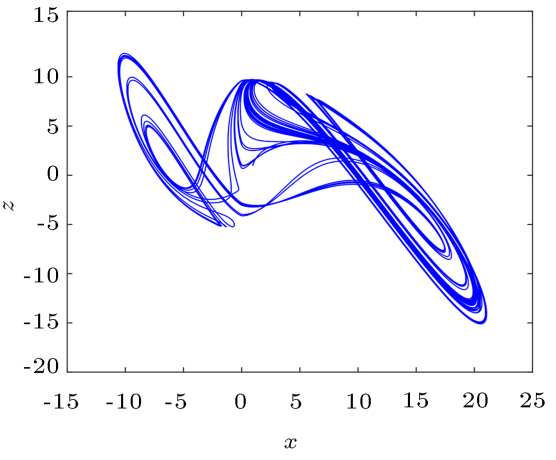

(b)

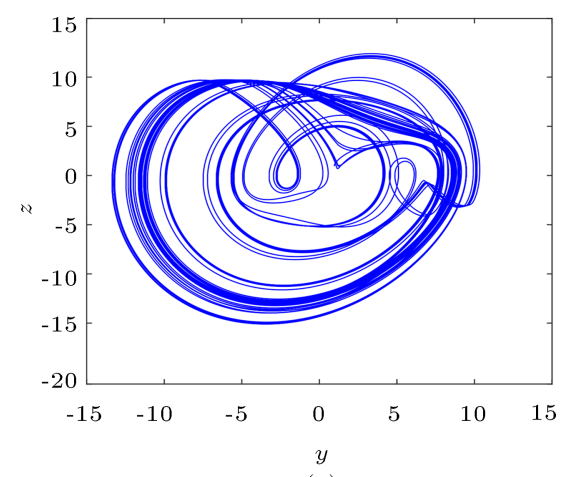

(c)

Figure 9. Three projections of the attractor of System (15) in (a) wx, (b) $z x$, and (c) $z y$ planes.

$w$ and $x$ are in the interval $[-50,50]$ and $[-15,25]$, respectively. $50 \mathrm{~V}$ and $25 \mathrm{~V}$ are not supported by the used common components. Thus, the tension should be less than $15 \mathrm{~V}$ to eliminate saturation problems. A linear transformation for the fractional-order System (15) is recommended to decrease amplitudes of the state variables. Let consider $x=2.5 u, y=2.5 v$, $z=2.5 \mathrm{~g}$, and $w=2.5 \mathrm{f}$. The adjusted system is described as follows:

$$
\left\{\begin{aligned}
D^{q} x= & y \\
D^{q} y= & z \\
D^{q} z= & a z-b y-2.5 c x^{2}+2.5 d|x| x \\
& +\frac{m}{\sqrt{2.5}} \sqrt{|x|} \operatorname{sgn}(x) \\
D^{q} w= & k y-h w-2.5 c x^{2}+2.5 d|x| x \\
& +\frac{m}{\sqrt{2.5}} \sqrt{|x|} \operatorname{sgn}(x)
\end{aligned}\right.
$$

The state variables $x$ and $w$ of System (16) are now included in the interval $[-15,15]$. Moreover, the fractional-order hyperchaotic Systems (15) and (16) have the same dynamics since the linear transformation does not change the physical properties of nonlinear systems.

\subsection{Circuit design of the fractional-order system}

Based on the previous work in [16], the electric circuit relative to System (16) with $q=1$ is described by the following equations:

$$
\left\{\begin{aligned}
\dot{x}= & \frac{1}{R_{1}^{\prime} C_{1}^{\prime}} y, \\
\dot{y}= & \frac{1}{R_{2}^{\prime} C_{2}^{\prime}} z, \\
\dot{z}= & -\frac{1}{R_{3}^{\prime} C_{3}^{\prime}} z-\frac{1}{R_{4}^{\prime} C_{3}^{\prime}} y+\frac{1}{R_{5}^{\prime} C_{3}^{\prime}} x^{2} \\
& -\frac{1}{R_{6}^{\prime} C_{3}^{\prime}}|x| x+\frac{1}{R_{7}^{\prime} C_{3}^{\prime}} \sqrt{|x|} \operatorname{sgn}(x) \\
\dot{w}= & -\frac{1}{R_{8}^{\prime} C_{4}^{\prime}} y-\frac{1}{R_{9}^{\prime} C_{4}^{\prime}} w+\frac{1}{R_{5}^{\prime} C_{4}^{\prime}} x^{2} \\
& -\frac{1}{R_{6}^{\prime} C_{4}^{\prime}}|x| x+\frac{1}{R_{7}^{\prime} C_{4}^{\prime}} \sqrt{|x|} \operatorname{sgn}(x)
\end{aligned}\right.
$$

where the resistor and capacitor values are:

$$
\begin{aligned}
& C_{1}^{\prime}=C_{2}^{\prime}=C_{3}^{\prime}=C_{4}^{\prime}=1 \mathrm{nF}, \quad R_{1}^{\prime}=R_{2}^{\prime}=100 \mathrm{k} \Omega, \\
& R_{3}^{\prime}=70 \mathrm{k} \Omega, \quad R_{4}^{\prime}=35 \mathrm{k} \Omega, \\
& R_{5}^{\prime}=100 \mathrm{k} \Omega, \quad R_{6}^{\prime}=35 \mathrm{k} \Omega, \\
& R_{7}^{\prime}=4.5 \mathrm{k} \Omega, \quad R_{8}^{\prime}=40 \mathrm{k} \Omega, \\
& R_{9}^{\prime}=10 \mathrm{k} \Omega .
\end{aligned}
$$

On the other hand, based on Eq. (8), the Laplace transformation of System (16) in $q=0.97$ is given as: 


$$
\left\{\begin{aligned}
\mathscr{L}\{x(t)\}= & \frac{1}{s^{0.97}} \mathscr{L}\{y(t)\}, \\
\mathscr{L}\{y(t)\}= & \frac{1}{s^{0.97}} \mathscr{L}\{z(t)\}, \\
\mathscr{L}\{z(t)\}= & \frac{1}{s^{0.97}} \mathscr{L}\left\{a z-b y-2.5 c x^{2}\right. \\
& \left.+2.5 d|x| x+\frac{m}{\sqrt{2.5}} \sqrt{|x|} \operatorname{sgn}(x)\right\}, \\
\mathscr{L}\{w(t)\}= & \frac{1}{s^{0.97}} \mathscr{L}\left\{k y-h w-2.5 c x^{2}\right. \\
& \left.+2.5 d|x| x+\frac{m}{\sqrt{2.5}} \sqrt{|x|} \operatorname{sgn}(x)\right\} .
\end{aligned}\right.
$$

Thus, based on Eq. (17), the integrator $q=1$ will be replaced by the integrator of fractional-order 0.97 . The product operation is realized with AD633 Multiplier. The root square function is realized by two operational amplifier LM741 and 7 resistors as described in [16]. Figure 10 describes the obtained circuit of System (16) designed by Multisim. For the oscillator circuit, all active devices (UA741 and AD633) are powered by $15 \mathrm{~V}$.
Several design considerations were taken into account to prevent degrading of the hyperchaotic behavior such as the adjustment of the electrical components relating to the fractional-order integrator. This is also related to the availability and standardization of electrical components in the market. After several tests, we opted for the following values for the fractional-order integrator:

$$
\begin{aligned}
& R_{1}=180 \mathrm{k} \Omega, \quad R_{2}=300 \mathrm{k} \Omega, \quad R_{3}=10 \mathrm{k} \Omega, \\
& C_{1}=0.5 \mathrm{nF}, \quad C_{2}=0.2 \mathrm{nF}, \quad C_{3}=0.1 \mathrm{nF} .
\end{aligned}
$$

Figure 11 represents the attractors of the designed circuit with Multisim software. The oscilloscope traces have the same forms as the attractors represented in Figure 9 (dark blue). It reflects the steady state. However, there is a minimal difference between the two

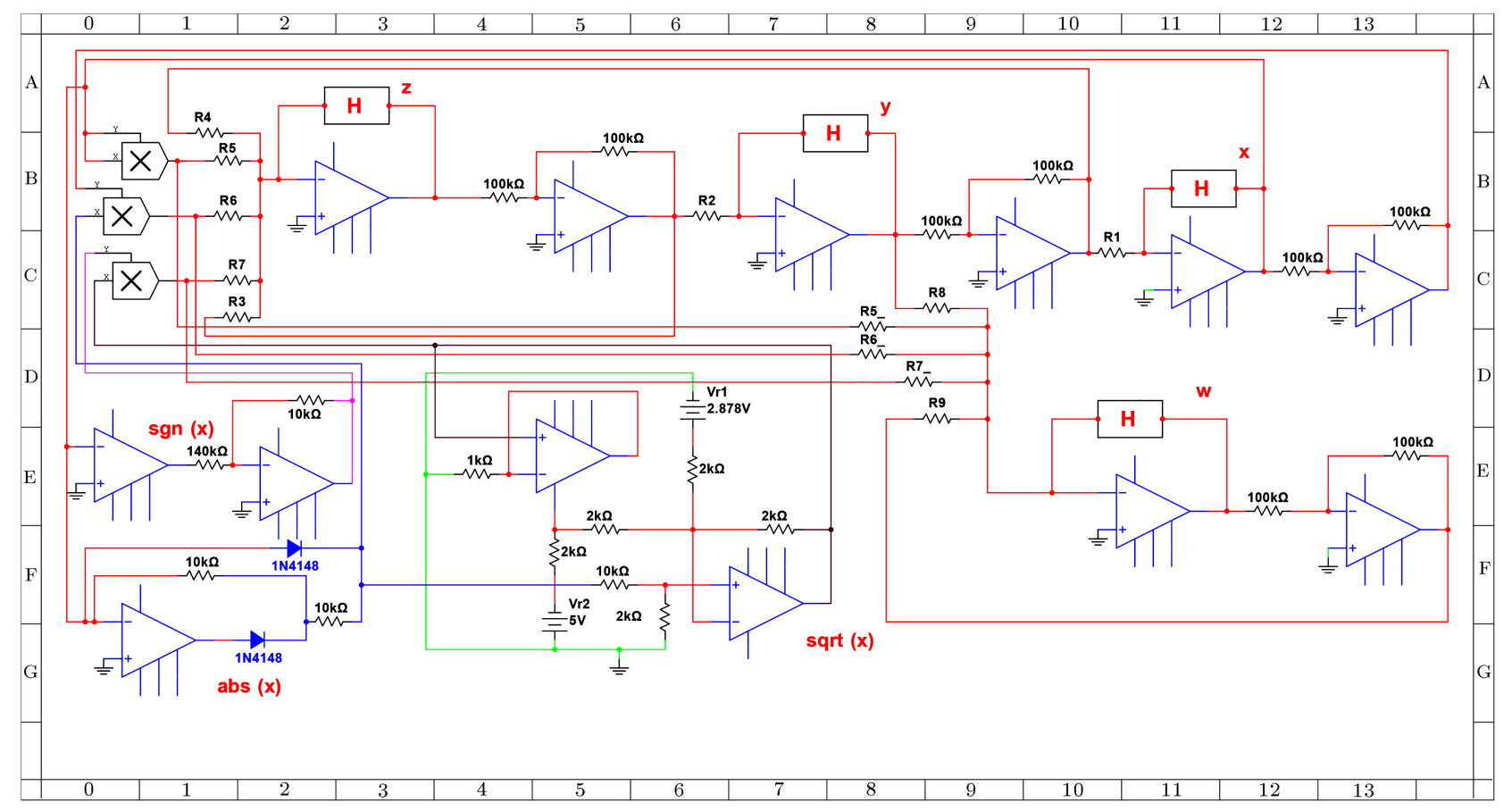

Figure 10. Design of the electrical circuit relative to System (16).
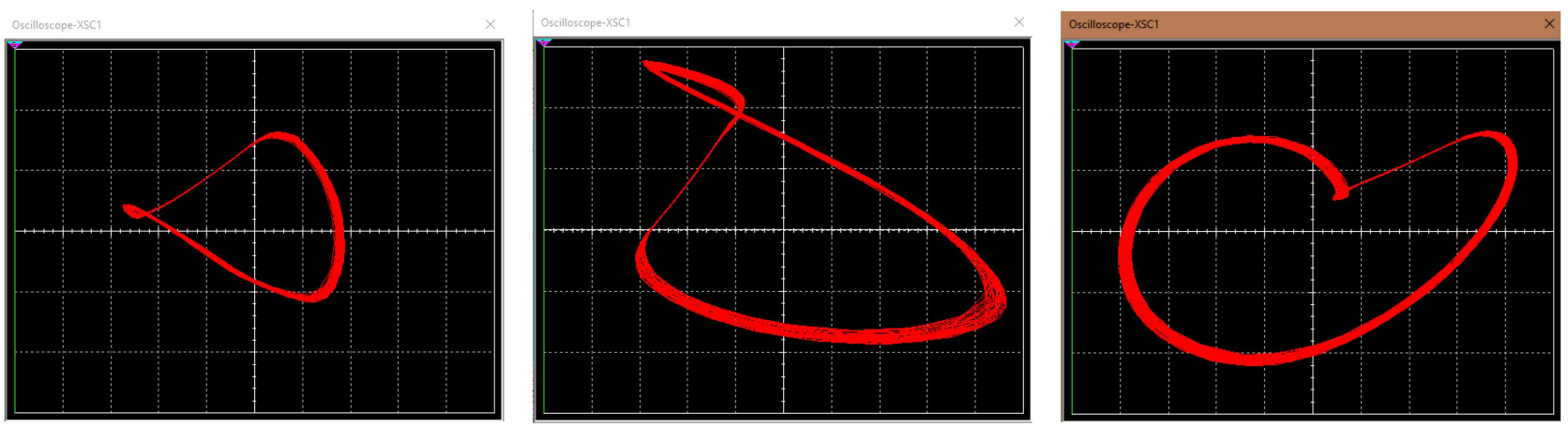

Figure 11. Strange attractors of the fractional-order System (16). 
attractors, which does not affect their structure but rather act on the rate of expansion and contraction over time.

\section{Conclusion}

In this paper, a hyperchaotic system with fractional terms and fractional-order derivatives was studied. The system featured different attractors such as equilibrium point, limit cycle, and hyperchaotic attractor. This study approximated the fractional derivative and designed the circuit of fractional-order integrator. Then, the circuit of our studied system was designed, which could show the same dynamics as the theoretical system. Declaration of interest: The authors declare that there is no conflict of interests regarding the publication of this paper.

\section{References}

1. Podlubny, I. "Fractional-order systems and pid controllers", IEEE Trans. Automat. Contr., 44(1), pp. 208-214 (1999).

2. Diethelm, K., The Analysis of Fractional Differential Equations: An Application-Oriented Exposition Using Differential Operators of Caputo Type, Springer-Verlag Berlin Heidelberg, Germany (2010).

3. Petras, I., Fractional-Order Nonlinear Systems: Modeling, Analysis and Simulation, Springer-Verlag Berlin Heidelberg, Germany (2011).

4. Vastarouchas, C., Psychalinos, C., and Elwakil, A.S. "Fractional-order model of a commercial ear simulator", 2018 IEEE Int. Symp. Circuits Syst., Florence, Italy, pp. 1-4 (2018).

5. Lassoued, A. and Boubaker, O. "On new chaotic and hyperchaotic systems: A literature survey", Nonlinear Anal-Model, 21(6), pp. 770-789 (2016).

6. Ran, J. "Discrete chaos in a novel two-dimensional fractional chaotic map", Adv. Differ. Equ., 2018, pp. 294:1-12 (2018).

7. Li, H., Liao, X., and Luo, M. "A novel non-equilibrium fractional-order chaotic system and its complete synchronization by circuit implementation", Nonlinear Dyn., 68(1-2), pp. 137-149 (2012).

8. Buscarino, A., Fortuna, L., Frasca, M., et al. "A chaotic circuit based on hewlett-packard memristor", Chaos, 22(2), pp. 023136:1-9 (2012).

9. Buscarino, A., Fortuna, L., and Frasca, M. "The jerk dynamics of chua's circuit", Int. J. Bifurc. Chaos, 24(06), pp. 1450085:1-10 (2014).

10. Bao, B., Wang, N., Chen, M., et al. "Inductorfree simplified chua's circuit only using two-op-ampbased realization", Nonlinear Dyn., 84(2), pp. 511-525 (2016).

11. Prakash, P., Singh, J.P., and Roy, B. "Fractionalorder memristor-based chaotic jerk system with no equilibrium point and its fractional-order backstepping control", IFAC-Papers OnLine, 51(1), pp. 1-6 (2018).

12. Vaidyanathan, S., Sambas, A., and Mamat, M. "Analysis, synchronisation and circuit implementation of a novel jerk chaotic system and its application for voice encryption", Int. J. Model. Identif. Control, 28(2), pp. 153-166 (2017).

13. Podlubny, I., Fractional Differential Equations: An Introduction to Fractional Derivatives, Fractional Differential Equations, to Methods of Their Solution and Some of Their Applications, Academic press, New York, USA (1998).

14. Jian-Bing, H. and Ling-Dong, Z. "Stability theorem and control of fractional systems", Acta. Phys. Sin., 62(24), pp. 240504:1-7 (2013).

15. Diethelm, K. and Ford, N.J. "Analysis of fractional differential equations", J. Math. Anal. Appl., 265(2), pp. 229-248 (2002).

16. Lassoued, A. and Boubaker, O. "Dynamic analysis and circuit design of a novel hyperchaotic system with fractional-order terms", Complexity, 2017, pp. 1-10 (2017).

17. Danca, M. and Kuznetsov, N. "Matlab code for lyapunov exponents of fractional-order systems", Int. J. Bifurc. Chaos, 28(05), pp. 1850067:1-14 (2018).

18. Charef, A., Sun, H., Tsao, Y., and Onaral, B. "Fractal system as represented by singularity function", IEEE Trans. Automat. Contr., 37(9), pp. 1465-1470 (1992).

19. Ahmad, W.M. and Sprott, J. "Chaos in fractionalorder autonomous nonlinear systems", Chaos Soliton. Fract., 16(2), pp. 339-351 (2003).

20. Ruo-Xun, Z. and Shi-Ping, Y. "Chaos in fractionalorder generalized lorenz system and its synchronization circuit simulation", Chin. Phys. B, 18(8), pp. 32953303 (2009).

\section{Biographies}

Abir Lassoued is a member of the Research Laboratory Energy, Robotics Control and Synchronization. She received the Engineering Diploma in Computer Science and Automatic Control from National Institute of Applied Sciences and Technology (INSAT) in 2013. She received the $\mathrm{PhD}$ in Computer Science from INSAT in 2019. Her research works are focused on chaos theory, nonlinear dynamics, fractional calculus, and chaos synchronization.

Fahimeh Nazarimehr was born in 1990 in Qazvin, Iran. She received the BSc degree in Biomedical Engineering at Shahed University in 2012 and the MSc degree at Sharif University of Technology in 2014, Iran. She received the $\mathrm{PhD}$ in Biomedical Engineering at Amirkabir University of Technology in 2018. Currently, she is a researcher at Biomedical Engineering Department at Amirkabir University of Technology; Iran. 
Her research interests include chaos theory and its application in biological signals and systems, nonlinear dynamics, bifurcation analysis, dynamical systems in neuroscience, feature extraction, and classification of signals.

Olfa Boubaker is a Full Professor of Control Systems Engineering and Robotics at the National Institute of Applied Sciences and Technology (INSAT) of University of Carthage, Tunisia. She was the Head of the Research Laboratory Energy, Robotics, Control and Optimization from 2016 to 2019. Professor Boubaker received the $\mathrm{PhD}$ degree in Electrical Engineering from National Engineering School of Tunis (ENIT) in 2000, and the Habilitation Universitaire from National Engineering School of Sfax (ENIS) in 2007. From 1997 to 2006, she was a Permanent Visitor of the
Laboratory for Analysis and Architecture of Systems (LAAS-CNRS), Toulouse, France. In 2019, she was a Plenary Speaker in Civil Aviation University of China, Taijin, China. Her research works focus on nonlinear systems, control theory, and Robotics. Professor Boubaker has developed a number of applications in several areas of sustainable development including the fields of secure communication, medical robotics, and ecologic sustainability. She is the author of more than 150 research works and has published 9 books ( 5 edited by Elsevier). Professor Boubaker has supervised 8 theses and 19 Master dissertations and mentored 70 engineer graduate projects in industry. Professor Boubaker is also associated with Elsevier as a Book Series Editor and serves as an Associate Editor and a Guest Editor in several international journals. 